\title{
Low insulin level is associated with aortic stiffness
}

\author{
Hiroshi Okada ${ }^{1}$, Michiaki Fukui ${ }^{1}$, Muhei Tanaka ${ }^{1}$, Satoshi Akabame ${ }^{2}$, Ki-ichiro Tomiyasu ${ }^{2}$, Koji Nakano ${ }^{2}$, \\ Masahiro Yamazaki ${ }^{1}$, Goji Hasegawa ${ }^{1}$, Yohei Oda $^{3}$ and Naoto Nakamura ${ }^{1}$
}

Recent studies have demonstrated that hyperinsulinemia is a risk factor for cardiovascular disease. The aim of this study was to evaluate the relationship between serum insulin level and the cardio-ankle vascular index (CAVI), which was developed as a marker of arterial stiffness. We performed a cross-sectional study of 260 consecutive and nondiabetic subjects with clinical suspicion of coronary heart disease. We measured CAVI in all subjects. A standard 75-g oral glucose tolerance test was performed, and plasma glucose and serum insulin levels were measured in venous blood collected at $0,30,60$ and $120 \mathrm{~min}$ after the test. Statistical analyses were conducted for four subgroups according to the insulin area under the concentration time curve (InsAUC). Mean CAVI and InsAUC were 8.7 and $109.5 \mu \mathrm{IU} \mathrm{ml}^{-1} \mathrm{~h}^{-1}$, respectively. Unadjusted analysis demonstrated that the InsAUC quartiles were significantly associated with CAVI $(P<0.0001)$, and the lowest InsAUC quartile $(P=0.001)$ had a lower glucose AUC. Analysis of covariance demonstrated that the lowest InsAUC quartile had the highest CAVI, and, after adjusting for several coronary risk factors, the highest InsAUC quartile had a higher CAVI than the second and third InsAUC quartiles $(P<0.0001)$. In conclusion, the lowest InsAUC quartile was related to CAVI, although the lowest InsAUC quartile maintained glucose homeostasis in this study population. Both hyperinsulinemia and low insulin level are independently associated with CAVI.

Hypertension Research (2011) 34, 336-340; doi:10.1038/hr.2010.234; published online 2 December 2010

Keywords: CAVI; hyperinsulinemia; low insulin level

\section{INTRODUCTION}

Hyperinsulinemia because of insulin resistance causes metabolic and hemodynamic abnormalities that are associated with a high cardiovascular risk. ${ }^{1,2}$ However, it is unknown whether low insulin level is associated with cardiovascular risk.

Arterial stiffness is associated with cardiovascular disease (CVD), and pulse wave velocity (PWV) and the cardio-ankle vascular index (CAVI) can be used to assess the degree of arterial stiffness. Classic PWV often has low reproducibility because it is difficult to measure and is strongly affected by blood pressure. CAVI has recently been developed as a marker of arterial stiffness that can be assessed simply and noninvasively. High values of either CAVI or PWV point to the progression of carotid and coronary arteriosclerosis. ${ }^{3}$ However, CAVI is independent of blood pressure and is therefore more easily reproduced than $\mathrm{PWV}^{4}$ In this study, we examined the relationship between serum insulin level and CAVI in nondiabetic subjects.

\section{METHODS}

\section{Subjects and study design}

We performed a cross-sectional study of 260 consecutive and nondiabetic subjects with clinical suspicion of coronary heart disease who visited the Department of Cardiology, Yamashiro Public Hospital (Kyoto, Japan), between April 2006 and November 2009. A standard 75-g oral glucose tolerance test was performed to evaluate each subject's glucose-load response; plasma glucose and serum insulin levels were measured in venous blood collected at $0,30,60$ and $120 \mathrm{~min}$ after the test. The product of glucose, insulin levels and time duration in the entire oral glucose tolerance test study was expressed as glucose area under the concentration time curve and insulin area under the concentration time curve (InsAUC). The subjects were divided into four subgroups according to fasting insulin quartiles and InsAUC quartiles, and we then examined the relationship between serum insulin levels and CAVI.

All subjects provided information about their demographic characteristics, medical history and medication usage. We measured CAVI and ankle brachial index (ABI) in all subjects. Body mass index was calculated as weight in kilograms divided by height in meters squared. Smoking was defined as current tobacco usage. Diabetic mellitus was defined as a fasting plasma glucose $\geqslant 126 \mathrm{mg}$ per $100 \mathrm{ml}$, 2-h postload glucose concentrations $\geqslant 200 \mathrm{mg}$ per $100 \mathrm{ml}$ or a current history of antidiabetic medication. All subjects were in stable clinical condition, and informed consent was obtained from all subjects under a protocol approved by the local investigational review board.

\section{Laboratory data}

In the morning, after an overnight fast, $10 \mathrm{ml}$ of venous blood was collected and used to measure creatinine, total cholesterol, triglyceride, fasting plasma glucose and fasting serum insulin levels. Hemoglobin Alc was assayed using highperformance liquid chromatography with a standard provided by the National Glycohemoglobin Standardization Program. Insulin resistance was expressed according to the homeostasis model assessment for insulin resistance. ${ }^{5}$

${ }^{1}$ Department of Endocrinology and Metabolism, Graduate School of Medical Science, Kyoto Prefectural University of Medicine, Kyoto, Japan; ${ }^{2}$ Department of Cardiology, Yamashiro Public Hospital, Kyoto, Japan and ${ }^{3}$ Department of Immunology and Inflammation, Graduate School of Medical Science, Kyoto Prefectural University of Medicine, Kyoto, Japan

Correspondence: Dr M Fukui, Department of Endocrinology and Metabolism, Graduate School of Medical Science, Kyoto Prefectural University of Medicine, 465 Kajii-cho, Kawaramachi-Hirokoji, Kamigyo-ku, Kyoto 602-8566, Japan.

E-mail: sayarinapm@hotmail.com

Received 9 June 2010; revised and accepted 9 September 2010; published online 2 December 2010 


\section{Measurements of ABI and CAVI}

The ABI was measured with a device that automatically and simultaneously measures blood pressures in both arms and ankles. ABI was calculated as the ratio of ankle systolic blood pressure (SBP) to arm SBP. CAVI was measured from an ECG, phonocardiogram, brachial artery waveform and ankle artery waveform and calculated using a specific algorithm (VaSera VS-1000; Fukuda Denshi, Tokyo, Japan). CAVI was calculated as follows: CAVI $=a\{(2 \rho / \Delta P)$ $\left.\times \ln (\mathrm{Ps} / \mathrm{Pd}) \mathrm{PWV}^{2}\right\}+\mathrm{b}$, where $\mathrm{Ps}$ is $\mathrm{SBP}$, Pd is diastolic blood pressure, $\mathrm{PWV}$ is pulse wave velocity, $\Delta \mathrm{P}$ is $\mathrm{Ps}-\mathrm{Pd}, \rho$ is blood density and a and $\mathrm{b}$ are constants. ${ }^{4}$ CAVI was evaluated as the larger value of the left or right side, and $\mathrm{ABI}$ was evaluated as the smaller value of the left or right side.

\section{Statistical analysis}

Categorical and continuous variables were compared among the groups by $\chi^{2}$ analysis and analysis of variance, respectively. Tukey-Kramer post hoc analysis was performed to determine significant differences among the groups. Analysis of covariance was used to assess differences among groups, adjusted for variable sets of covariates. Model 1 adjusted for age, sex and body mass index; model 2 adjusted for model 1 plus smoking status, antihypertensive drugs, nitrates, statins, SBP, heart rate, total cholesterol, triglyceride, blood urea nitrogen (BUN), creatinine, uric acid, high sensitivity C-reactive protein (hs-CRP), hemoglobin, hemoglobin Alc and fasting plasma glucose; and model 3 adjusted for model 2 plus ischemic heart disease, stroke and arteriosclerosis obliterans.

Continuous variables are presented as means \pm 1 s.d., and categorical variables are presented as numbers (percentages). A $P$-value of $<0.05$ was considered statistically significant. The statistical analyses were performed using the JMP version 8.0 software (SAS Institute, Cary, NC, USA).

\section{RESULTS}

Table 1 shows characteristics of all subjects (170 men, 90 women, average age $69.3 \pm 10.3$ years, age range $34-90$ years). Mean CAVI and InsAUC were 8.7 and $109.5 \mu \mathrm{IU} \mathrm{ml}^{-1} \mathrm{~h}^{-1}$, respectively. Table 2 summarizes crude and unadjusted risk factor levels according to fasting insulin and InsAUC quartiles. The fasting insulin quartiles were significantly associated with age $(P=0.0005)$, sex $(P=0.0005)$, body mass index $(P<0.0001)$, SBP $(P=0.02)$, heart rate $(P=0.04)$, total cholesterol $(P=0.04)$, triglyceride $(P<0.0001)$, BUN $(P=0.0008)$, uric acid $(\mathrm{P}=0.004)$, homeostasis model assessment for insulin resistance $(P<0.0001)$ and CAVI $(P=0.0344)$ (analysis of variance). The InsAUC quartiles were significantly associated with age $(P<0.0001)$, arteriosclerosis obliterans $(P=0.01)$, body mass index $(P<0.0001)$, heart rate $(P=0.01)$, triglyceride $(P=0.01)$, uric acid $(P=0.04)$, hs-CRP $(P=0.05)$, homeostasis model assessment for insulin resistance $(P<0.0001)$, ABI $(P=0.0002)$ and CAVI $(P<0.0001)$. Sex, stroke, smoking status, antihypertensive drugs, nitrates, statins, ischemic heart disease, SBP, total cholesterol, BUN, creatinine, hemoglobin and hemoglobin A1c did not differ among the InsAUC quartiles. The lowest InsAUC quartile had a lower glucose area under the concentration time curve. As shown in Table 3, CAVI did not differ among the fasting insulin quartiles (analysis of covariance); however, the lowest InsAUC quartile had the highest CAVI, and the highest InsAUC quartile had a higher CAVI than the second and third InsAUC quartiles $(P<0.0001)$ (analysis of covariance).

\section{DISCUSSION}

Hyperinsulinemia is a known risk factor for CVD. The burden of hyperinsulinemia because of insulin resistance in diabetic patients is widely known, but few studies have examined the risks associated with low insulin levels in nondiabetic patients. We evaluated the involvement of low insulin level as a cardiovascular risk. The degree of aortic stiffness is associated with the development of metabolic and hemodynamic abnormalities, which involve a high cardiovascular risk. ${ }^{4}$
Table 1 Participant characteristics

\begin{tabular}{|c|c|}
\hline$n$ & 260 \\
\hline Age (years) & $69.3(10.3)$ \\
\hline Male (\%) & $170(65.4)$ \\
\hline $\mathrm{BMI}\left(\mathrm{kg} \mathrm{m}^{-2}\right)$ & $23.6(3.7)$ \\
\hline Smoking (\%) & $26(10.0)$ \\
\hline Antihypertensive drugs (\%) & $75(28.8 \%$ \\
\hline Nitrates (\%) & $58(22.3 \%$ \\
\hline Statins (\%) & $111(42.7 \%$ \\
\hline IHD (\%) & 31 (11.9) \\
\hline ASO (\%) & $16(6.2)$ \\
\hline Stroke (\%) & $7(2.7)$ \\
\hline $\mathrm{SBP}(\mathrm{mm} \mathrm{Hg})$ & $137.6(20.5)$ \\
\hline Heart rate (b.p.m.) & $66.7(11.5)$ \\
\hline Total cholesterol (mg per $100 \mathrm{ml}$ ) & $180.8(35.7)$ \\
\hline Triglyceride (mg per 100 ml) & $122.8(61.5)$ \\
\hline BUN (mg per $100 \mathrm{ml}$ ) & $18.6(4.5)$ \\
\hline Creatinine (mg per $100 \mathrm{ml}$ ) & $0.87(0.62)$ \\
\hline Uric acid (mg per 100 ml) & $5.6(1.5)$ \\
\hline hs-CRP (mg per 100 ml) & $0.11(0.19)$ \\
\hline Hemoglobin (g per 100 ml) & $12.9(1.9)$ \\
\hline $\mathrm{HbAlc}(\%)$ & $5.5(0.3)$ \\
\hline HOMA-IR & $1.4(1.0)$ \\
\hline $\mathrm{ABI}$ & $1.1(0.1)$ \\
\hline CAVI & $8.7(1.3)$ \\
\hline \multicolumn{2}{|l|}{ Glucose (mg per $100 \mathrm{ml})$} \\
\hline $0 \mathrm{~min}$ & $89.7(8.5)$ \\
\hline $30 \mathrm{~min}$ & $149.4(25.4)$ \\
\hline $60 \mathrm{~min}$ & $161.9(41.4)$ \\
\hline $120 \mathrm{~min}$ & $130.7(32.0)$ \\
\hline AUC & $283.9(48.7)$ \\
\hline \multicolumn{2}{|l|}{ Insulin $\left(\mu \mid \cup \mathrm{ml}^{-1}\right)$} \\
\hline $0 \mathrm{~min}$ & $6.1(4.0)$ \\
\hline $30 \mathrm{~min}$ & $53.4(44.2)$ \\
\hline $60 \mathrm{~min}$ & $66.1(56.5)$ \\
\hline $120 \mathrm{~min}$ & $63.4(72.2)$ \\
\hline 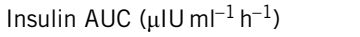 & $109.5(86.2)$ \\
\hline
\end{tabular}

Abbreviations: $A B I$, ankle brachial index; ASO, arteriosclerosis obliterans; AUC, area under the concentration time curve; BMI, body mass index (calculated as weight in kilograms divided by height in meters squared); CAVI, cardio-ankle vascular index; IHD, ischemic heart disease; HbAlc, glycated hemoglobin Alc; HOMA-IR, homeostasis model assessment for insulin resistance; SBP, systolic blood pressure.

Data are expressed as mean (s.d.) or absolute number (percent).

In recent years, PWV or CAVI has been considered the gold standard method for the assessment of aortic stiffness. Charalambos et al. ${ }^{6}$ suggested that aortic stiffness determined by PWV is a strong predictor of future cardiovascular events and all-cause mortality. However, classic PWV is technically difficult to measure and has low reproducibility. Although brachial-ankle PWV is easy to measure and has high reproducibility, it is affected strongly by blood pressure. CAVI is easy to measure, is less affected by blood pressure and reflects arterial stiffness. A recent study proposed that the intra-observer correlation coefficient was 0.82 for right CAVI, 0.87 for left CAVI and 0.85 for mean CAVI; these findings indicate that this index is reliable and reproducible. ${ }^{7}$ Therefore, CAVI is more useful than PWV and has recently become widely used for the evaluation of arterial stiffness. In this study, we obtained similar results using the average CAVI (right and left CAVI) instead of the higher CAVI (data not shown).

Moreover, we examined the correlation between CAVI and indicators of aortic stiffness such as carotid intima-media thickness (IMT) and plaque score. The relationships between CAVI and mean IMT, max IMT and plaque score measured by ultrasonography were further 
Table 2 Unadjusted mean characteristics according to fasting insulin and insulin area under the concentration time curve levels (ANOVA)

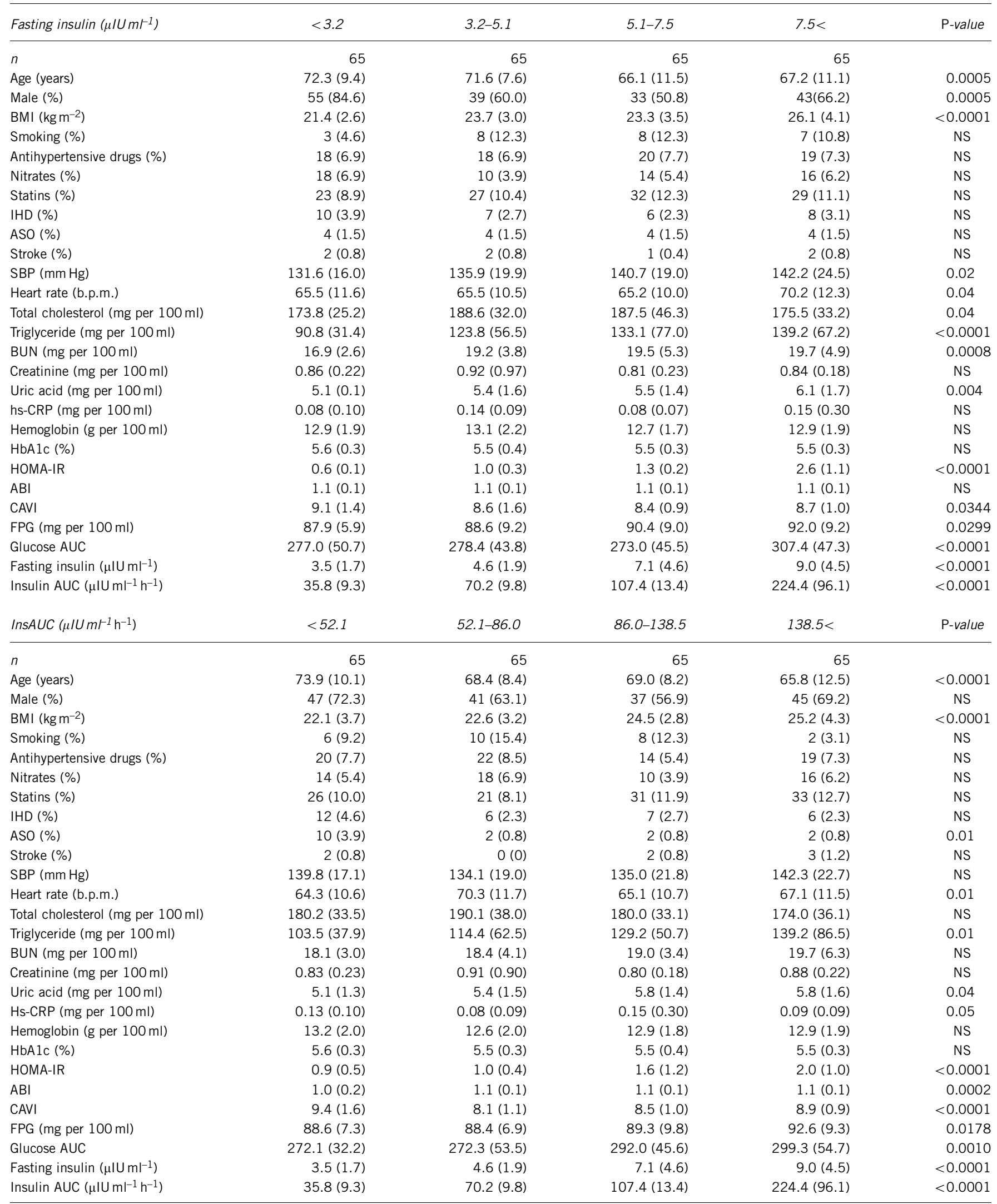

Abbreviations: ABI, ankle brachial index; ANOVA, analysis of variance; ASO, arteriosclerosis obliterans; AUC, area under the concentration time curve; BMI, body mass index (calculated as weight in kilograms divided by height in meters squared); CAVI, cardio-ankle vascular index; FPG, fasting plasma glucose; HbAlc, glycated hemoglobin A1c; HOMA-IR, homeostasis model assessment for insulin resistance; IHD, ischemic heart disease; NS, not significant; SBP, systolic blood pressure.

$P$-value of $<0.05$ was considered to be statistically significant. Data are expressed as mean (s.d.) or absolute number (percent). 
Table 3 Adjusted least square mean CAVI according to insulin area under the concentration time curve levels (ANCOVA)

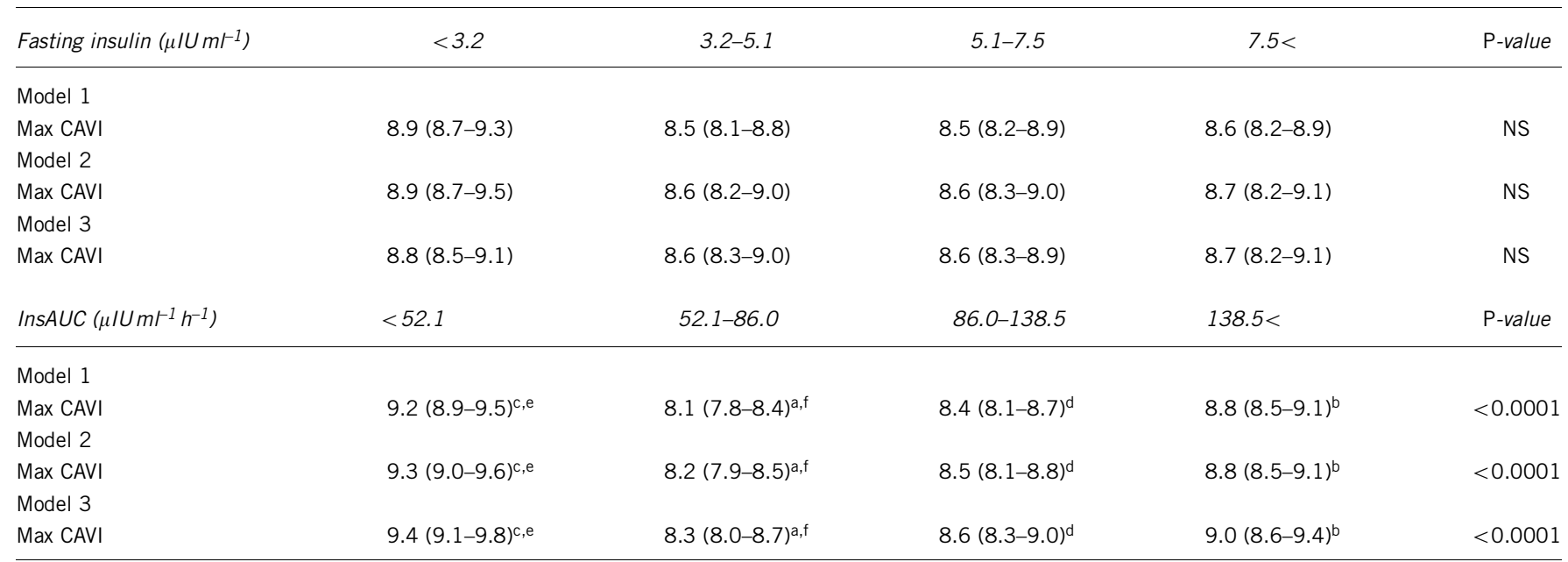

Abbreviations: ANCOVA, analysis of covariance; CAVI, cardio-ankle vascular index; InsAUC, insulin area under the concentration time curve; NS, not significant.

The post hoc analysis indicated a significant difference between these two quartiles.

The post hoc analysis indicated a significant difference between these two quartiles.
$\mathrm{a}, \mathrm{b} P<0.05 ; \mathrm{c}, \mathrm{d} P<0.005 ;{ }^{\mathrm{e}, \mathrm{f}} P<0.0005$. Model 1 adjusted for age, sex and body mass index; model 2 adjusted for model 1 plus smoking status, antihypertensive drugs, nitrates, statins, systolic blood pressure, heart rate, total cholesterol, triglyceride, BUN, creatinine, uric acid, hs-CRP, hemoglobin, HbAlc and fasting plasma glucose; model 3 adjusted for model 2 plus ischemic heart disease, stroke and arteriosclerosis obliterans; data are expressed as mean ( $95 \%$ confidence interval).

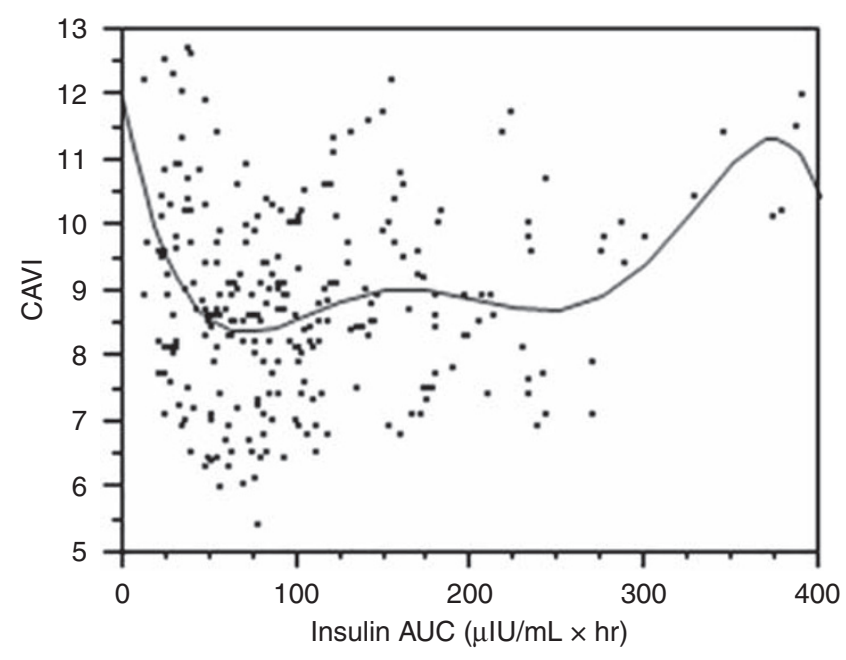

Figure 1 Fitting-curve presented relation between InSAUC and CAVI.

investigated in a subgroup of patients $(n=167)$. A positive correlation was found between CAVI and mean IMT $(r=0.281, P<0.001)$, max IMT $(r=0.213, P<0.001)$ and plaque score $(r=0.277, P<0.001)$.

The lower InsAUC quartile was associated with a lower glucose level than the other quartiles. However, the relationship between CAVI and the InsAUC was not linear. The relationship between CAVI and the InsAUC was a U-shaped curve, as shown Figure 1. The U-shaped curve indicated that the lowest InsAUC quartile was the specific cluster. Similar results were obtained from the division in either three or five groups of fasting insulin and InsAUC (data not shown). We therefore concluded that the division of the subjects into four groups was reasonable.

Subjects in the lowest InsAUC quartile were older than those in other quartiles. Indeed, previous studies suggested that age was an important risk factor for CVD. ${ }^{8}$ A total of 98 patients (37.7\%) were over 75 years of age. Therefore, age-adjustment is very important in this study. The lowest InsAUC quartile was associated with CAVI, even after adjustment for age by analysis of covariance.
Increasing vascular stiffness induced by endothelial dysfunction is a developmental process of CVD. Some studies suggested that endothelial dysfunction, which is related to the pathogenesis of CVD, is caused by oxidative stress. In addition, vascular stiffness is related to superoxide generation in the vessel wall. ${ }^{9}$ Insulin has several direct vascular effects that contribute to either cell injury or cell protection. Ricci et al. ${ }^{10}$ suggested that if a cell is subjected to insulin withdrawal, superoxide generation is altered. As insulin is a cytoprotective factor, cell survival could be significantly affected by insulin concentrations. Insulin deficiency leads to an increase in reactive oxygen species production and results in oxidative mitochondrial DNA damage. In other words, a deficiency of insulin could cause oxidative stress and cell apoptosis and lead to aortic stiffness.

In addition, insulin has other vasculoprotective roles. Nitric oxide, which preserves endothelial conditions, is protective and inhibits platelet aggregation, monocyte adhesion, local inflammation, oxidation, vascular smooth muscle cell (VSMC) growth and migration. ${ }^{11}$ Insulin dilates vessels by stimulating nitric oxide synthesis and its release from the endothelium. ${ }^{12}$ This stimulation results in VSMC relaxation and the inhibition of atherosclerosis. Moreover, insulin may decrease the contractile response of VSMC to vasoactive agents by decreasing the intracellular calcium concentration. ${ }^{12}$ Cecilia et al. ${ }^{13}$ suggested that insulin had effects on animal model VSMC calcification in vitro and that insulin attenuates VSMC calcification in a dosedependent manner. Some studies postulated that a state of insufficient cell insulinization might be the atherogenic factor. ${ }^{14-16}$ Either hypoinsulinemia or hyperinsulinemia may affect the dysfunction of one cell type that has a significant role in atherogenesis. Taking these findings together, it seems plausible that low insulin level is also related to CAVI.

In this study, we demonstrated the relationship between insulin level and CAVI. In addition, insulin levels were correlated with homeostasis model assessment for insulin resistance in Table 2. Therefore, the relationship indicated that not only insulin resistance but also low insulin level (that is, high insulin sensitivity) might be linked to CAVI. These facts suggest that the process of atherosclerosis may begin in the state of insufficient insulinization, whether caused by insulin resistance or low insulin level (that is, high insulin sensitivity). 


\section{Study limitations}

This study is limited by its cross-sectional design, which does not allow us to determine causality. Most study subjects were similar in the sense that they were highly motivated to assess their coronary heart disease risk and had symptoms or clinical signs of coronary heart disease at the time of diagnosis. Large prospective trials are needed to better assess the relationship between low insulin level and CAVI.

Wildman et al. ${ }^{17}$ suggested that menopause is associated with subclinical atherosclerosis. Therefore, it is important to know how many postmenopausal women were included in our study. Unfortunately, we had no exact data on how many postmenopausal women were included, but almost all the female subjects (97.8\%) were over 50 years of age. We can assume that these women were postmenopausal and that the effect of menopause was low.

\section{CONCLUSIONS}

The lowest InsAUC quartile was related to CAVI, although the lowest InsAUC quartile maintained glucose homeostasis in subjects with clinical suspicion of coronary heart disease. Both hyperinsulinemia and low insulin level are independently associated with CAVI.

1 Shea S, Ma S, Liu K, Preethi S, Jacobs Jr DR, Wu C, Saad MF, Szklo M. Insulin resistance, metabolic syndrome, and subclinical atherosclerosis: the Multi-Ethnic Study of Atherosclerosis (MESA). Diabetes Care 2007; 30: 2951-2956.

2 Theuma P, Fonseca VA. Inflammation, insulin resistance, and atherosclerosis. Metab Syndr Relat Disord 2004; 2: 105-113.

3 Izuhara M, Shioji K, Kadota S, Baba O, Takeuchi Y, Uegaito T, Mutsuo S, Matsuda M. Relationship of cardio-ankle vascular index (CAVI) to carotid and coronary arteriosclerosis. Circ J 2008; 72: 1762-1767.
4 Shirai K, Utino J, Otsuka K, Takata M. A novel blood pressure-independent arterial wall stiffness parameter; cardio-ankle vascular index (CAVI). J Atheroscler Thromb 2006; 13: 101-107.

5 Hanyu O, Yoshida J, Abe E, Hirayama S, Miyake K, Aizawa Y, Miida T. High-sensitivity CRP reflects insulin resistance in smokers. J Atheroscler Thromb 2009; 16: 560-567.

6 Vlachopoulos C, Aznaouridis K, Stefanadis C. Prediction of cardiovascular events and all-cause mortality with arterial stiffness: a systematic review and meta-analysis. J Am Coll Cardiol 2010; 55: 1318-1327.

7 Huang CL, Chen MF, Jeng JS, Lin LY, Wang WL, Feng MH, Liau CS, Hwang BS, Lee YT, Su TC. Postchallenge hyperglycaemic spike associate with arterial stiffness. Int J Clin Pract 2007; 61: 397-402.

8 Ramadan MM, Mahfouz EM, Gomaa GF, El-Diasty TA, Alldawi L, Ikrar T, Limin D, Kodama M, Aizawa Y. Evaluation of coronary calcium score by multidetector computed tomography in relation to endothelial function and inflammatory markers in asymptomatic individuals. Circ J 2008; 72: 778-785.

9 Delles C, Zimmerli LU, McGrane DJ, Koh-Tan CH, Pathi VL, McKay AJ, Steedman T, Dargie HJ, Hamilton CA, Dominiczak AF. Vascular stiffness is related to superoxide generation in the vessel wall. J Hypertens 2008; 26: 946-955.

10 Ricci C, Pastukh V, Mozaffari M, Schaffer SW. Insulin withdrawal induces apoptosis via a free radical-mediated mechanism. Can J Physiol Pharmacol 2007; 85: 455-464.

11 Hsueh WA, Quiñones MJ. Role of endothelial dysfunction in insulin resistance. Am J Cardiol 2003; 92: 10J-17J.

12 Mikhail N, Tuck ML. Insulin and the vasculature. Curr Hypertens Rep 2000; 2: 148-153.

13 Wang CC, Sorribas V, Sharma G, Levi M, Draznin B. Insulin attenuates vascular smooth muscle calcification but increases vascular smooth muscle cell phosphate transport. Atherosclerosis 2007; 195: e65-e75.

14 Trovati M, Anfossi G, Cavalot F, Massucco P, Mularoni E, Emanuelli G. Insulin directly reduces platelet sensitivity to aggregating agents: studies in vitro and in vivo. Diabetes 1988; 37: 780-786.

15 Burzacca S, Ponziani MC, Massucco P, Mattiello L, Piretto V, Cavalot F, Anfossi G. Impaired insulin-induced platelet anti-aggregating effect in obesity and obese NIDDM patients. Diabetes 1995; 44: 1318-1322.

16 Steinberg HO, Brechtel G, Johnson A, Fineberg N, Baron AD. Insulin-mediated skeletal muscle vasodilation is nitric oxide dependent: a novel action of insulin to increase nitric oxide release. J Clin Invest 1994; 94: 1172-1179.

17 Wildman RP, Schott LL, Brockwell S, Kuller LH, Sutton-Tyrrell K. A dietary and exercise intervention slows menopause-associated progression of subclinical atherosclerosis as measured by intima-media thickness of the carotid arteries. J Am Coll Cardiol 2004; 44: 579-585. 